\title{
Contribution of wind shear to the entrainment process of the atmospheric convective boundary layer
}

\author{
Anran Li, Wenfeng Gao*, and Tao Liu
}

School of Energy and Environment Sciences, Yunnan Normal University, Kunming, China

\author{
Article history: \\ Received 24 September 2019 \\ Revised 10 December 2019 \\ Accepted 11 February 2020 \\ Keywords: \\ Convective boundary layer, Stratified \\ flow, The entrainment layer, Wind \\ shear

\section{Citation:} \\ Li, A., W. Gao, and T. Liu, 2020: \\ Contribution of wind shear to \\ the entrainment process of the \\ atmospheric convective boundary \\ layer. Terr. Atmos. Ocean. \\ Sci., 31, 351-358, doi: 10.3319/ \\ TAO.2020.02.11.01
}

\begin{abstract}
Wind shear induced by the velocity difference has an important effect on the stably stratified turbulent flow in the atmospheric convective boundary layer (CBL). Cases with different velocity difference are conducted by Large-eddy simulation (LES) to reveal the contribution of shear effect to entrainment. The result shows that the shear-generated turbulent kinetic energy (TKE) in the entrainment layer is partially consumed by the negative buoyancy, which increases the entrainment flux minimum and enhances the entrainment flux ratio in the range of $0.188-0.352$. Based on the hypothesis of the independence of the shear-generated TKE and buoyancygenerated $T K E$, an approach for the fraction of the shear-generated $T K E$ available for entrainment is proposed, and the averaged fraction reaches $38.5 \%$, but the evolution tends to increase with the enlargement of velocity difference.
\end{abstract}

\section{INTRODUCTION}

Due to the velocity difference in the entrainment layer between the mixed layer and the free atmosphere layer, the wind shear enhances entrainment processes and plays a key role in the mater and energy exchange of the stratified flow in the atmospheric CBL, which makes the CBL's main characteristics and the turbulence statistics significantly different from that of the shear-free CBL (Christian and Wakimoto 1989; Nilsson et al. 2012). The study of contribution of shear effect on CBL is helpful to establish the entrainment velocity scale and to predict the height and thickness of the entrainment layer, which is of great significance for atmospheric science and environmental engineering, for example, improving the accuracy of numerical weather prediction, predicting the distribution of pollutants in the atmosphere.

Most studies on the sheared entrainment in CBL were carried out in the framework of bulk models, and the firstorder jump model (FOM) was considered more close to the actual conditions, in which the linear variation of variables

\footnotetext{
* Corresponding author

E-mail: solar@ynnu.edu.cn
}

with height in CBL is assumed to adequately capture the entrainment process in sheared CBL (Conzemius and Fedorovich 2007; Huang et al. 2011; Gentine et al. 2015). The idealized vertical profiles of the potential temperature, wind velocity and buoyancy flux in the FOM are illustrated in Fig. 1.

Fedorovich et al. (2001a, b) and Pino et al. (2003) found that the shear effect in the surface layer or entrainment layer promotes the occurrence of entrainment and the growth of entrainment layer through numerical simulation and experiments. The presence of horizontal wind in convective situations increases the entrainment flux and modifies the convection pattern. Moreover, the entrainment flux can be further enhanced. Analysis of TKE budgets indicates that turbulence in the entrainment layer capping the cloud is produced locally by wind shear and is dynamically decoupled from turbulence driven by convective circulations across CBL (Kopec et al. 2016; Haghshenas and Mellado 2019).

The essential problem relates to characterization of the shear effect used for entrainment is to propose an appropriate index. Many studies derived a parameterization for 
the entrainment flux ratio in the framework of FOM, stating that the entrainment flux is enlarged by wind shear (Kim et al. 2006; Pino and Arellano 2008). Regrettably, as the entrainment flux in the FOM is always larger than that derived from the LES heat flux profiles, they found the parameterization somewhat overestimated the entrainment flux ratio, as well as the entrainment heat flux. In order to improve the accuracy of model prediction, Sun and Xu (2009) modified some coefficients in this scheme suitable for their results with LES data. The entrainment flux ratio can only reflect the promotion of entrainment, and cannot separately distinguish the effect of shear and buoyancy on entrainment.

Another parameterized method to evaluate the contribution of shear to entrainment is based on the vertical integration of TKE transport equation (Pino et al. 2003; Kim et al. 2006; Pino and Arellano 2008). Pino and De Arellano (2008) used local momentum fluxes and velocity jumps to represent the shear production rate of TKE to evaluate the various contributions to the TKE budgets, and the influence of shear on entrainment was shown in the vertical distribution of the various terms of the TKE budgets. By the scaling analysis, this parameterization is an improvement of the one advanced by Pino et al. (2003) and incorporates the physical arguments of each TKE term not discussed by Kim et al. (2006) or Pino and Arellano (2008).

A parameterization method was derived from the $T K E$ budgets for a well-developed CBL under the condition of linearly sheared velocity with a zero value at the surface (Liu et al. 2016a, b). Results suggested that about $43 \%$ of the shear-produced TKE in the entrainment layer is available for entrainment, while the shear-produced TKE in the mixed layer and surface layer has little effect on entrainment.

This study takes the stably stratified turbulent flow in a horizontally homogeneous atmospheric CBL as the object to study the effect of wind shear caused by different velocity difference in the entrainment layer on the entrainment process. Firstly, we analyze the influence of wind shear on the entrainment layer thickness of CBL with different boundary conditions by LES method. Subsequently, the entrainment flux ratio is analyzed and compared as a quantitative index of entrainment in the sheared and shear-free CBLs. Finally, an attempt is made to propose a parametric approach to calculate the fraction of shear-generated TKE in the entrainment layer available for entrainment.

\section{LES EXPERIMENTS AND OUTPUT}

\subsection{Numerical Setup}

This study carries out LES codes described by Moeng (1984) and refined by Moeng and Wyngaard (1988) for studying the evolution of the CBL, which was also adopted in the parameterization study of sheared CBL by Moeng and Sullivan (1994), Kim et al. (2003, 2006), and Botnick and Fedorovich (2008).

Eight cases with varied boundary conditions (velocity difference, buoyancy flux and potential temperature gradient) are performed by LES numerical method in a domain of $6000 \mathrm{~m} \times 6000 \mathrm{~m} \times 2000 \mathrm{~m}$ with an aspect ratio of 6 bigger than the empirical value 4.5 (Hadfield et al. 1992). The grid intervals take $\Delta X=\Delta Y=25 \mathrm{~m}$ and $\Delta Z=20 \mathrm{~m}$, and in the vicinity of the surface sublayer and the entrainment layer with large gradient, the grid intervals vary steadily from 3 to $25 \mathrm{~m}$ so as to capture certain features of the turbulence.

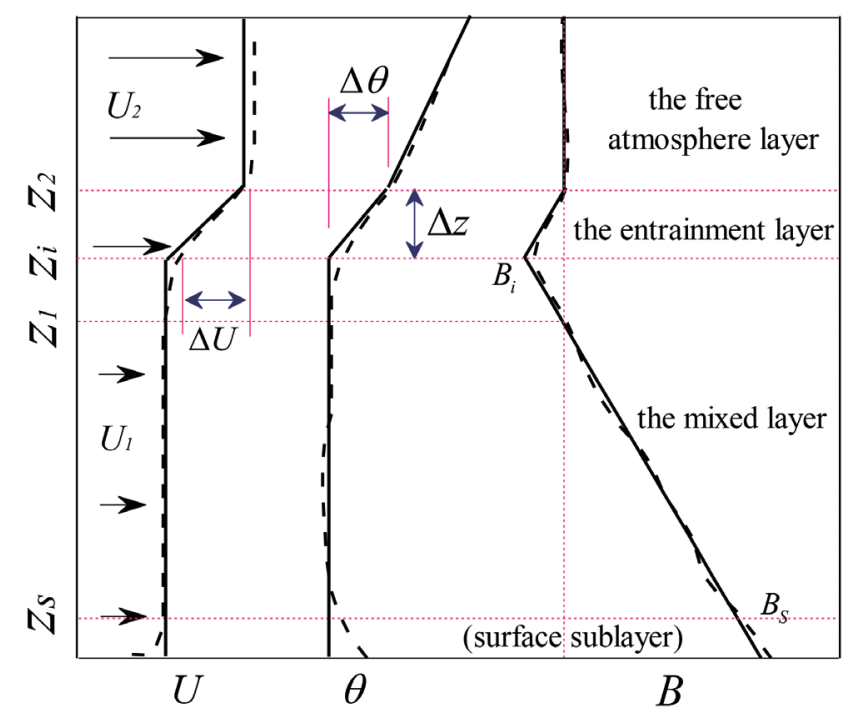

Fig. 1. Schematic of the horizontal velocity $U$, the potential temperature $\theta$, and buoyancy flux $B$ in the atmospheric CBL. Dashed lines stand for the realistic horizontally averaged profiles; solid lines represent their counterparts in the "first-order model" (FOM) (Betts 1973). $\Delta U$ and $\Delta \theta$ are the jump value of the horizontal velocity and the potential temperature across the entrainment zone; $\Delta z$ is the thickness of the entrainment zone; $B_{i}$ and $B_{s}$ are the buoyancy flux at the entrainment layer and the surface, respectively. 
The numerical domain adopts a periodic lateral boundary conditions and a "rigid-lid" upper boundary where the vertical fluxes of physical parameters such as mass, momentum and heat are zero. The entrainment layer height $z_{i}$ is initialized to $1000 \mathrm{~m}$, below which the potential temperature $\theta$ is set to $300 \mathrm{~K}$, and above which with the gradient $\Gamma_{\theta}$ of 0.003 $-0.006 \mathrm{~K} \cdot \mathrm{m}^{-1}$. The surface buoyancy flux $B_{s}$ is set to 0.05 $0.1 \mathrm{~K} \cdot \mathrm{ms}^{-1}$. The main data are shown in Table 1 .

The unsteady calculation time step of all simulations is $0.2 \mathrm{~s}$ and the quasi-steady calculation time satisfies the experiential requirement of $t>6 t_{*}$ (Cai and Chen 1997), where the time scale $t_{*}$ is $t_{*}=z_{i} / w_{*}$, ensuring the TKE does not change significantly with time and the flow in a stable development stage at the end of the calculation.

Three-dimensional data volumes are archived from the final stage of the simulations to gather statistics and to present flow visualization. The ensemble means and turbulence statistics shown here are the result of spatial averaging, that is, at a fixed $z$ location averaging over a $x-y$ plane.

\subsection{Thickness of the Entrainment Layer}

The flow field of this study is simplified based on the FOM. The height of entrainment layer $z_{i}$ corresponds to the buoyancy flux minimum (the negative maximum), and the upper limit of entrainment layer $z_{2}$ takes the corresponding height at zero of buoyancy flux in the free atmosphere layer. Specially pointed out that due to the influence of large-scale eddies in the upper layer, the fluctuation of $z_{2}$ should not be ignored, and the corresponding height of $10 \% B_{i}$ is generally permitted (Fedorovich et al. 2004); the height of underlying surface $z_{s}$ is $90 \% B_{s}$; the thickness of entrainment layer is $\Delta z=z_{2}-z_{i}$.

The surficial buoyancy flux is a major turbulence production mechanism, and a stronger surficial buoyancy flux enlarges the temperature difference between the thermals (some hot air masses in the surface sublayer) and the ambience, which determines the depth of the thermals penetrating into the free atmosphere and decisively impacts the entrainment development. Compared with each row of profiles in Fig. 2, the enhancement of $B_{s}$ from 0.05 to $0.1 \mathrm{~K} \mathrm{~ms}^{-1}$ expands $\Delta z$ by $10-70 \mathrm{~m}$, and the influence is particularly greater in weak stratified environment (cases with smaller $\Gamma_{\theta}$ ), which is consistent with the analytic conclusion of shear-free CBL. In addition, $z_{i}$ also increases correspondingly under the same conditions of $\Gamma_{\theta}$ and $\Delta U$, indicating the promotion of entrainment.

Due to the thermals behavior in the upper part of the entrainment zone $\left(z_{i}-z_{2}\right)$ dominantly affected by negative buoyancy, the inhibition of entrainment by the potential temperature gradient of free atmosphere is significantly obvious through comparing with column 3 vs 1,4 vs 2 of Fig. 2. $\Delta z$ and $z_{i}$ are significantly shrink with increase of $\Gamma_{\theta}$, especially in the cases with weak surficial buoyancy flux.
Kim et al. (2003) and Pino et al. (2003) considered that the vertical shear stress of horizontal wind destroyed the stability of Kelvin-Helmholtz wave at the interface and enhanced entrainment. The thickness of the entrainment zone is directly related to the strength of velocity difference under the same $B_{s}$ and $\Gamma_{\theta}$ conditions, referring to column 2 vs 1 and column 4 vs 3 of Fig. 2, especially in the cases with weak inversion strength ( $\left(\operatorname{smaller} \Gamma_{\theta}\right.$ ) where $\Delta z$ increases more remarkably. For example, when $\Delta U$ changes from 1.5 to $3 \mathrm{~m} \mathrm{~s}^{-1}, \Delta z$ of $\mathrm{A} 12$ raises $79 \mathrm{~m}$ over that of $\mathrm{A} 11$, while A22 only $18 \mathrm{~m}$ over A21, revealing that the turbulence intensity in the entrainment zone with weak inversion strength is small, and the shear generated TKE consumed by the negative buoyancy in the entrainment zone increases accordingly, which makes the thickness increase more.

\section{ENTRAINMENT FLUX RATIO}

\subsection{TKE Transport Equation}

In the horizontally homogeneous CBL, TKE transport equation in the vertical direction can be described as:

$$
\begin{aligned}
\frac{\partial \bar{e}}{\partial t}= & -\left(\overline{u^{\prime} w^{\prime}} \frac{\partial U}{\partial z}+\overline{v^{\prime} w^{\prime}} \frac{\partial V}{\partial z}\right) \\
& +\frac{g}{\bar{\theta}_{0}} \overline{w^{\prime} \theta^{\prime}}-\frac{\partial \overline{w^{\prime} e}}{\partial z}-\frac{1}{\rho_{0}} \frac{\partial \overline{w^{\prime} p^{\prime}}}{\partial z}-\varepsilon
\end{aligned}
$$

where $\bar{e}$ is the averaged TKE, $\rho_{0}$ and $\theta_{0}$ are the ambient density and potential temperature, $p$ is the pressure, $\varepsilon$ is the turbulent viscous dissipation rate, and $u, v$, and $w$ are the velocity components, respectively. Overbars denote the horizontal averages and primes indicate deviations from the horizontal average.

In order to investigate the contribution of wind shear to the entrainment process of the stably stratified turbulent flow, we adopt the process partitioning method (VanZanten et al. 1999; Conzemius and Fedorovich 2006) to calculate the TKE budgets, which includes the generation and the dissipation of TKE. Because shear promotes entrainment, and enlarges the buoyancy flux minimum in the entrainment layer, the integral change of the net buoyancy flux in the entrainment layer compared with shear-free CBL is usually used to quantify the contribution of shear to entrainment. To integrate $T K E$ transport equation vertically over the depth of the CBL, we can obtain:

$$
\begin{aligned}
\frac{d}{d t} \int_{0}^{z_{i}} \bar{e} d z & \\
= & \underbrace{\left(u_{m} \tau_{x \cdot s}+v_{m} \tau_{y \cdot s}\right)+\frac{1}{2}\left(\Delta U^{2}+\Delta V^{2}\right) \frac{d z_{i}}{d t}+\frac{z_{i}}{2} B_{s}}_{\text {generation }} \\
& \underbrace{-\frac{z_{i}}{2} \Delta b \frac{d z_{i}}{d t}-\int_{0}^{z_{i}} \varepsilon d z}_{\text {dissipation }}+\underbrace{\int_{0}^{z_{0}}\left(-\frac{\partial \overline{w^{\prime} e^{\prime}}}{\partial z}-\frac{1}{\rho_{0}} \frac{\partial \overline{w^{\prime} p}}{\partial z}\right) d z}_{\text {transporation }}
\end{aligned}
$$


Where $\Delta U, \Delta V$, and $\Delta b$ are the change of the $x$ - , $y$-component velocity and buoyancy force across the entrainment layer, and $\tau_{x \cdot s}$ and $\tau_{y \cdot s}$ are the shear stresses at the surface, respectively.

It can be inferred from Eq. (2) that TKE generation is composed of the distribution of the shear stress in the surface sublayer $\left(u_{m} \tau_{x \cdot s}+v_{m} \tau_{y \cdot s}\right)$ and in the entrainment layer $\frac{1}{2}\left(\Delta U^{2}+\Delta V^{2}\right) \frac{d z_{i}}{d t}$, and the distribution of the thermal buoyancy importing from the surface $\frac{z_{i}}{2} B_{s}$. The dissipation of $T K E$ consists of the negative buoyancy contribution in the entrainment layer $-\frac{z_{i} \Delta b}{2} \frac{d z_{i}}{d t}$ and the turbulent viscous dissipation $-\int_{0}^{z_{i}} \varepsilon d z$. The transportation term just influences the vertical distribution of TKE, and normally so small under typical atmospheric CBL that can be ignored in the hereafter analysis (Fedorovich et al. 2004).
The shear-generated TKE near the surface must pass upward through the whole lower layer to affect the entrainment process, and the vertical motion of the thermals can effectively relax the mean wind turbulence in the mixed layer, making it be dissipated within a short distance. Moreover, Conzemius and Fedorovich (2006) considered that shear in the surface had little effect on entrainment compared with the shear effect in entrainment layer. Hence, the shear-generated TKE in the surface is not considered in this paper when studying the effect of shear on the entrainment.

\subsection{Entrainment Flux Ratio for Sheared CBL}

In the shear-free CBL, the buoyancy flux inputted from the surface is the only source of the turbulence generation.

Table 1. Dataset of the numerical cases with different velocity difference $\Delta U$.

\begin{tabular}{c|ccccc}
\hline \multirow{2}{*}{ Case } & $\boldsymbol{B}_{S}$ & $\boldsymbol{U}_{\mathbf{1}}$ & $\boldsymbol{U}_{\mathbf{2}}$ & $\boldsymbol{\Gamma}_{\boldsymbol{\theta}}$ & $\boldsymbol{\Delta} \boldsymbol{U}$ \\
\cline { 2 - 6 } & $\mathbf{K ~ m s}^{-\mathbf{1}}$ & $\mathbf{m ~ s}^{-\mathbf{1}}$ & $\mathbf{m ~ s}^{-\mathbf{1}}$ & $\mathbf{K ~ m}^{-\mathbf{1}}$ & $\mathbf{m ~ s}^{-\mathbf{1}}$ \\
\hline $\mathrm{A} 11$ & 0.05 & 3 & 4.5 & 0.006 & 1.5 \\
$\mathrm{~A} 12$ & 0.05 & 3 & 6 & 0.006 & 3 \\
$\mathrm{~A} 21$ & 0.05 & 3 & 4.5 & 0.015 & 1.5 \\
$\mathrm{~A} 22$ & 0.05 & 3 & 6 & 0.015 & 3 \\
$\mathrm{~B} 11$ & 0.10 & 3 & 4.5 & 0.006 & 1.5 \\
$\mathrm{~B} 12$ & 0.10 & 3 & 6 & 0.006 & 3 \\
$\mathrm{~B} 21$ & 0.10 & 3 & 4.5 & 0.015 & 1.5 \\
$\mathrm{~B} 22$ & 0.10 & 3 & 6 & 0.015 & 3 \\
\hline
\end{tabular}
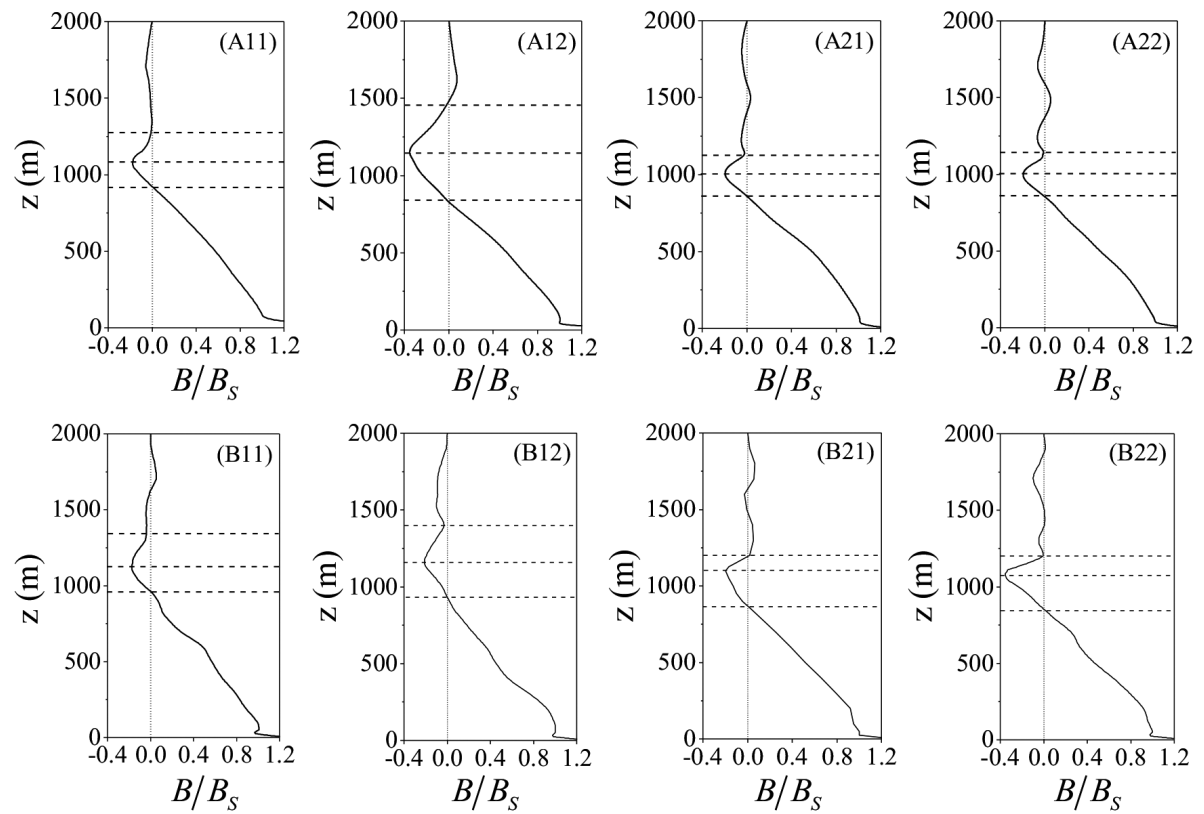

Fig. 2. Evolution of the buoyancy flux profiles for the numerical cases in Table 1 . The buoyancy flux is normalized by $B_{s}$. The limits of the entrainment zone are indicated by dashed lines. 
Some of the buoyancy-generated TKE is dissipated by the entrainment process and the rest is converted into heat by dissipation of the viscous. The fraction of the first part to the inputted surface buoyancy flux is defined as entrainment flux ratio $A_{f}$, i.e., $A_{f}=\left(\Delta b \frac{d z_{i}}{d t}\right) / B_{s}=\frac{B_{i}}{B_{s}}$. A large number of experiments and observations take the ratio of approximately 0.2 (Stull 1973; Pino et al. 2003; Fedorovich et al. 2004).

However, in the sheared CBL, the shear-generated $T K E$ cannot be completely dissipated by viscous, and the remaining is dissipated by a part of the negative buoyancy $\left(\Delta b \frac{d z_{i}}{d t}\right)$ in the entrainment layer, that is, the shear effect promotes the entrainment process and enlarges the minimum of the buoyancy flux in the entrainment layer, which results in an increase of the entrainment flux ratio., i.e., $A_{f}=\left(\Delta b \frac{d z_{i}}{d t}\right) / B_{s}>0.2$. The schematic profiles are illustrated in Fig. 3. Therefore, $A_{f}$ can be regarded as an indicator of shear effect on entrainment and can be used to quantitatively analyze the fraction of shear-generated TKE in the entrainment layer available for entrainment.

In our study, with the increase of the velocity difference, the shear-generated TKE is partly used to promote entrainment, and the value of $A_{f}$ shown in Fig. 2 is in the range of $0.188-0.385$ with an average value of 0.252 , greater than the value of shear-free CBL, especially in the cases with big buoyancy flux, small temperature gradient and great velocity difference.

\section{THE FRACTION OF SHEAR-GENERATED TKE AVAILABLE FOR ENTRAINMET}

The buoyancy flux is partitioned by method developed by Manins and Turner (1978) and further explained by Van-
Zanten et al. (1999) and Conzemius and Fedorovich (2006), which assumed that the buoyancy flux changes from TKE generation to dissipation at a certain height, below which the vertical integral of buoyancy flux $B$ is used to calculate buoyancy-generated TKE, and above which the vertical integral of $B$ is the TKE dissipated by negative buoyancy.

Based on the FOM of CBL (illustrated in Fig. 1), the buoyancy flux $B$ reads:

$$
B\left(\frac{z}{z_{i}}\right)= \begin{cases}B_{s}\left[\left(1-\frac{z}{z_{i}}\right)-A_{f} \frac{z}{z_{i}}\right] & 0 \leq \frac{z}{z_{i}} \leq 1 \\ -A_{f} B_{s}\left(1-\frac{\frac{z}{z_{i}}-1}{\frac{z_{2}}{z_{i}}-1}\right) & 1 \leq \frac{z}{z_{i}} \leq \frac{z_{2}}{z_{i}} \\ 0 & \frac{z_{2}}{z_{i}} \leq \frac{z}{z_{i}} \leq \infty\end{cases}
$$

Therefore, TKE budget dissipated by the entrainment process is:

$$
\begin{aligned}
& \int_{0}^{\infty} B d z \\
& \quad=\int_{0}^{z_{i}} B_{s}\left[\left(1-\frac{z}{z_{i}}\right)-A_{f} \frac{z}{z_{i}}\right] d z-\int_{z_{i}}^{z_{2}} A_{f} B_{s}\left(1-\frac{\frac{z}{z_{i}}-1}{\frac{z_{2}}{z_{i}}-1}\right) d z \\
& \quad=\frac{B_{s} z_{i}}{2}\left[1-\left(1+\frac{\delta z}{z_{i}}\right) A_{f}\right]
\end{aligned}
$$

For the shear-free CBL, $A_{f}=0.2$, thus,

$$
\int_{0}^{\infty} B d z=0.38 B_{s} z_{i}
$$

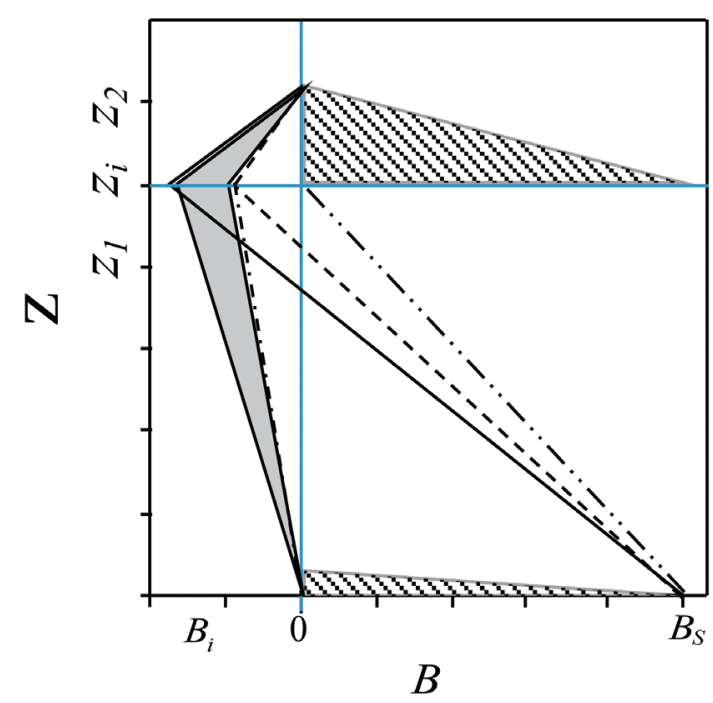

Fig. 3. The vertical profiles of the buoyancy flux of CBL. Solid lines represent sheared CBL buoyancy flux, dotted lines represent the shear-free CBL buoyancy flux, dash-dotted lines indicate the total negative buoyancy dissipation (entrainment consumption), the shaded area indicates the buoyancy flux of the sheared CBL is more destructed than that of CBL, double-dotted lines represent buoyancy generation, and the skewed area is shear generated TKE. 
Assuming that the shear-generated TKE doesn't affect the buoyancy-generated TKE and vice versa, buoyancygenerated TKE and its distribution available for entrainment in the sheared CBL remain unchanged relative to the shearfree CBL. Therefore, the vertical integral of the buoyancy flux variation can be used to calculate the amount of TKE dissipated by entrainment in the presence of shear, and then the fraction of shear-generated TKE available for entrainment can be calculated.

Therefore, for the sheared CBL, the fraction of sheargenerated TKE available for entrainment $\gamma_{p}$ can be written as:

$$
\begin{aligned}
\gamma_{p} & =\frac{\int_{0}^{z_{2}} B d z-0.38 B_{s} z_{i}}{\int_{z_{s}}^{z_{2}}\left(-\overline{u^{\prime} w^{\prime}} \frac{\partial U}{\partial z}-\overline{v^{\prime} w^{\prime}} \frac{\partial V}{\partial z}\right) d z} \\
& =\frac{\frac{B_{s} z_{i}}{2}\left[1-\left(1+\frac{\delta z}{z_{i}}\right) A_{f}\right]-0.38 B_{s} z_{i}}{\int_{z_{s}}^{z_{2}}\left(-\overline{u^{\prime} w} \frac{\partial U}{\partial z}-\overline{v^{\prime} w} \frac{\partial V}{\partial z}\right) d z} \\
& =\frac{\left(\frac{B_{s} z_{i}}{2}-\frac{B_{i} z_{2}}{2}\right)-0.38 B_{s} z_{i}}{\int_{z_{s}}^{z_{2}}\left(-\overline{u^{\prime} w^{\prime}} \frac{\partial U}{\partial z}-\overline{v^{\prime} w^{\prime}} \frac{\partial V}{\partial z}\right) d z} \\
& \approx \frac{\frac{B_{s} z_{2}}{2}\left[\left(A_{f}\right)_{S C B L}-\left(A_{f}\right)_{C B L}\right]}{\int_{z_{s}}^{z_{2}}\left(-\overline{u^{\prime} w} \frac{\partial U}{\partial z}-\bar{v}^{\prime} w^{\prime} \frac{\partial V}{\partial z}\right) d z}
\end{aligned}
$$

Here $\left(A_{f}\right)_{C B L}$ and $\left(A_{f}\right)_{S C B L}$ are the entrainment flux ratio in the shear-free CBL and sheared CBL, respectively.

Pino and Arellano (2008) adopted the thickness of the entrainment layer $\Delta z$ as the characteristic scale, and gave the shear stresses as following:

$-\overline{u^{\prime} w} \frac{\partial U}{\partial z}-\overline{v^{\prime} w} \frac{\partial V}{\partial z}=\left.C_{M} u_{*}\right|_{i} ^{2}\left(\frac{\Delta U}{\Delta z}\right)$ where, $C_{M}=1.15$, and considering the anisotropy of the flow, the friction velocity of the entrainment layer is

$\left.u_{*}\right|_{i}=\left(\left.\overline{u^{\prime} w^{\prime}}\right|_{i} ^{2}+\left.\overline{v^{\prime} w^{\prime}}\right|_{i} ^{2}\right)^{0.25}$

Thus, with Eqs. (7) and (8), Eq. (6) can be written as:

$\gamma_{p} \approx \frac{\frac{B_{s} z_{2}}{2}\left[\left(A_{f}\right)_{S C B L}-\left(A_{f}\right)_{C B L}\right]}{\int_{z_{s}}^{z_{s}}\left[C_{M}\left(\left.\overline{u^{\prime} w^{\prime}}\right|_{i} ^{2}+\left.\overline{v^{\prime} w^{\prime}}\right|_{i} ^{2}\right)^{0.5}\left(\frac{\Delta U}{\Delta z}\right)\right] d z}$

In the numerical cases of this study, the result of shearfree CBL is taken as the comparison criterion, and $\gamma_{p}=0$. The calculated results through Eq. (9) are shown in Fig. 4. The shear-generated TKE is dissipated by negative buoyancy, and $\gamma_{p}$ increases with the enhancement of shear. The average value of $\gamma_{p}$ is $38.5 \%$, which approximates to that of Liu et al. (2016a, b) of 43\%. The conclusion of Liu et al. (2016a, b) got from the sheared CBL with a greater wind shear. Therefore, whether $\gamma_{p}$ is close to a constant value needs to be further study in a wider boundary condition.

\section{CONCLUSION}

By studying the stratified CBL with different velocity difference, the shear-generated TKE in the entrainment layer, $\frac{1}{2}\left(\Delta U^{2}+\Delta V^{2}\right) \frac{d z_{i}}{d t}$, is partly dissipated by the molecular viscous, and the remainder is dissipated by the negative buoyancy in the entrainment layer, $-\frac{z_{i} \Delta b}{2} \frac{d z_{i}}{d t}$, which makes the minimum of the buoyancy flux larger with increase of the velocity difference, as well as the entrainment flux ratio $A_{f}$. With the enhancement of the velocity difference, more

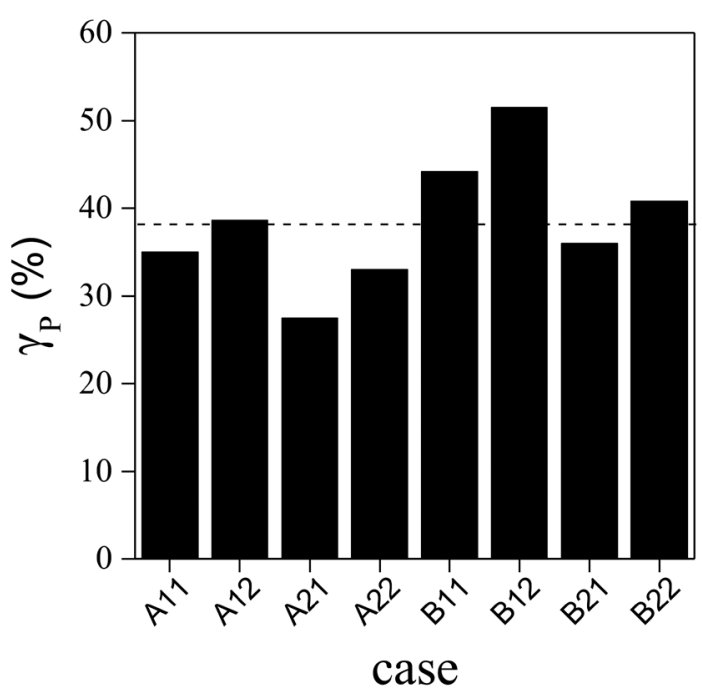

Fig. 4. The fraction of shear-generated TKE available for entrainment in the numerical cases. The dashed line denotes the average value. 
upper air is entrained downward, and the averaged $A_{f}$ reaches 0.252 bigger than that of shear-free CBL.

This study assumes that the buoyancy-generated TKE is independent of the existence of shear, and establishes the equation for the fraction of shear-generated TKE available for entrainment, $\gamma_{p}$, by separately calculating the generation and dissipation of TKE. As for eight numerical cases in this study, $\gamma_{p}$ increases with the enhancement of wind velocity difference, which indicates the shear promoting effect on the entrainment process. The averaged $\gamma_{p}$ reaches $38.5 \%$, which approximates to the result of Liu et al. $(2016 \mathrm{a}, \mathrm{b})$ of $43 \%$. Whether $\gamma_{p}$ tends to be stable in a wider boundary condition needs to be further studied.

Acknowledgements This work was supported by the National Natural Science Foundation of China (Grant No. 11662021 and No. 51866016), and got help from Computational Fluid Dynamics Laboratory of the Solar Energy Research Institute of Yunnan Normal University. The authors thank the anonymous reviewers, whose comments greatly helped to improve the manuscript.

\section{REFERENCES}

Betts, A. K., 1973: Non-precipitating cumulus convection and its parameterization. Q. J. R. Meteorol. Soc., 99, 178-196, doi: 10.1002/qj.49709941915. [Link]

Botnick, A. M. and E. Fedorovich, 2008: Large eddy simulation of atmospheric convective boundary layer with realistic environmental forcings. In: Meyers, J., B. J. Geurts, and P. Sagaut (Eds.), Quality and Reliability of Large-Eddy Simulations, Ercoftac Series, Vol. 12, Springer, Dordrecht, 193-204, doi: 10.1007/978-14020-8578-9_16. [Link]

Cai, X. and J. Chen, 1997: Large eddy simulation of bubblelike structures in the convective boundary layer. Chin. J. Atmos. Sci., 21, 223-230, doi: 10.3878/j.issn.10069895.1997.02.11. (in Chinese) [Link]

Christian, T. W. and R. M. Wakimoto, 1989: The relationship between radar reflectivities and clouds associated with horizontal roll convection on $8 \mathrm{Au}-$ gust 1982. Mon. Weather Rev., 117, 1530-1544, doi: 10.1175/1520-0493(1989)117<1530:trbrra>2.0.co;2. [Link]

Conzemius, R. J. and E. Fedorovich, 2006: Dynamics of sheared convective boundary layer entrainment. Part I: Methodological background and large-eddy simulations. J. Atmos. Sci., 63, 1151-1178, doi: 10.1175/ JAS3691.1. [Link]

Conzemius, R. J. and E. Fedorovich, 2007: Bulk models of the sheared convective boundary layer: Evaluation through large eddy simulations. J. Atmos. Sci., 64, 786807, doi: 10.1175/JAS3870.1. [Link]

Fedorovich, E., F. T. M. Nieuwstadt, and R. Kaiser, 2001a:
Numerical and laboratory study of a horizontally evolving convective boundary layer. Part I: Transition regimes and development of the mixed layer. J. Atmos. Sci., 58, 70-86, doi: 10.1175/1520-0469(2001)058 $<0070$ :nalsoa $>2.0 . c o ; 2$. [Link]

Fedorovich, E., F. T. M. Nieuwstadt, and R. Kaiser, 2001b: Numerical and laboratory study of horizontally evolving convective boundary layer. Part II: Effects of elevated wind shear and surface roughness. J. Atmos. Sci., 58, 546-560, doi: 10.1175/1520-0469(2001)058 $<0546$ :nalsoh>2.0.co;2. [Link]

Fedorovich, E., R. Conzemius, and D. Mironov, 2004: Convective Entrainment into a Shear-Free, Linearly Stratified Atmosphere: Bulk Models Reevaluated through Large Eddy Simulations. J. Atmos. Sci., 61, 281-295, doi: 10.1175/1520-0469(2004)061<0281:CEIASL >2 . $0 . \mathrm{CO} ; 2$. [Link]

Gentine, P., G. Bellon, and C. C. van Heerwaarden, 2015: A closer look at boundary layer inversion in largeeddy simulations and bulk models: Buoyancy-driven case. J. Atmos. Sci., 72, 728-749, doi: 10.1175/JASD-13-0377.1. [Link]

Hadfield, M. G., W. R. Cotton, and R. A. Pielke, 1992: Large-eddy simulations of thermally forced circulations in the convective boundary layer. Part II: The effect of changes in wavelength and wind speed. Bound.-Layer Meteor., 58, 307-327, doi: 10.1007/ BF00120235. [Link]

Haghshenas, A. and J. P. Mellado, 2019: Characterization of wind-shear effects on entrainment in a convective boundary layer. J. Fluid Mech., 858, 145-183, doi: 10.1017/jfm.2018.761. [Link]

Huang, J., X. Lee, and E. G. Patton, 2011: Entrainment and budgets of heat, water vapor, and carbon dioxide in a convective boundary layer driven by timevarying forcing. J. Geophys. Res., 116, D06308, doi: 10.1029/2010JD014938. [Link]

Kim, S.-W., S.-U. Park, and C.-H. Moeng, 2003: Entrainment processes in the convective boundary layer with varying wind shear. Bound.-Layer Meteor., 108, 221245, doi: 10.1023/a:1024170229293. [Link]

Kim, S.-W., S.-U. Park, D. Pino, and J. V.-G. de Arellano, 2006: Parameterization of entrainment in a sheared convective boundary layer using a first-order jump model. Bound.-Layer Meteor., 120, 455-475, doi: 10.1007/s10546-006-9067-3. [Link]

Kopec, M. K., S.P. Malinowski, and Z.P. Piotrowski, 2016: Effects of wind shear and radiative cooling on the stratocumulus-topped boundary layer. Q. J. R. Meteorol. Soc., 142, 3222-3233, doi: 10.1002/qj.2903. [Link]

Liu, P., J. Sun, and L. Shen, 2016a: Parameterization of sheared entrainment in a well-developed CBL. Part I: Evaluation of the scheme through large-eddy simulations. Adv. Atmos. Sci., 33, 1171-1184, doi: 10.1007/ 
s00376-016-5208-x. [Link]

Liu, P., J. Sun, and L. Shen, 2016b: Parameterization of sheared entrainment in a well-developed CBL. Part II: A simple model for predicting the growth rate of the CBL. Adv. Atmos. Sci., 33, 1185-1198, doi: 10.1007/ s00376-016-5209-9. [Link]

Manins, P. C. and J. S. Turner, 1978: The relation between the flux ratio and energy ratio in convectively mixed layers. Q. J. R. Meteorol. Soc., 104, 39-44, doi: 10.1002/qj.49710443904. [Link]

Moeng, C.-H., 1984: A large-eddy-simulation model for the study of planetary boundary-layer turbulence. J. Atmos. Sci., 41, 2052-2062, doi: 10.1175/1520-0469(1984)041<2052:alesmf>2.0 .co;2. [Link]

Moeng, C.-H. and P. P. Sullivan, 1994: A comparison of shear- and buoyancy-driven planetary boundary layer flows. J. Atmos. Sci., 51, 999-1022, doi: 10.1175/1520-0469(1994)051<0999:ACOSAB>2.0. CO;2. [Link]

Moeng, C.-H. and J. C. Wyngaard, 1988: Spectral analysis of large-eddy simulations of the convective boundary layer. J. Atmos. Sci., 45, 3573-3587, doi: 10.1175/1520-0469(1988)045<3573: saoles >2.0.co;2. [Link]

Nilsson, E. O., A. Rutgersson, A.-S. Smedman, and P. P.
Sullivan, 2012: Convective boundary-layer structure in the presence of wind-following swell. Q.J.R. Meteorol.Soc., 138, 1476-1489, doi: 10.1002/qj.1898. [Link]

Pino, D. and J. V.-G. De Arellano, 2008: Effects of shear in the convective boundary layer: Analysis of the turbulent kinetic energy budget. Acta Geophys., 56, 167 193, doi: 10.2478/s11600-007-0037-z. [Link]

Pino, D., J. V.-G. de Arellano, and P. G. Duynkerke, 2003: The contribution of shear to the evolution of a convective boundary layer. J. Atmos. Sci., 60, 1913-1926, doi: 10.1175/1520-0469(2003)060<1913:TCOSTT $>2.0$. $\mathrm{CO} ; 2$. [Link $]$

Stull, R. B., 1973: Inversion rise model based on penetrative convection. J. Atmos. Sci., 30, 1092-1099, doi: 10.1175/1520-0469(1973)030<1092:IRMBOP>2.0. $\mathrm{CO} ; 2 .[\underline{\mathrm{Link}}]$

Sun, J. and Q. Xu, 2009: Parameterization of sheared convective entrainment in the first-order jump model: Evaluation through large-eddy simulation. Bound.Layer Meteor., 132, 279-288, doi: 10.1007/s10546009-9394-2. [Link]

VanZanten, M. C., P. G. Duynkerke, and J. W. M. Cuijpers, 1999: Entrainment parameterization in convective boundary layers. J. Atmos. Sci., 56, 813-828, doi: 10.1175/1520-0469(1999)056<0813:epicbl>2.0.co;2. [Link] 